\title{
Solvability conditions for some systems of nonlinear non-Fredholm elliptic equations
}

\author{
Vitali Vougalter \\ Department of Mathematics and Applied Mathematics, University of Cape Town \\ Private Bag, Rondebosch 7701, South Africa \\ e-mail: Vitali.Vougalter@uct.ac.za
}

\begin{abstract}
We establish the existence and uniqueness of standing solitary wave-like solutions in $H^{2}$ for certain systems of nonlocal nonlinear equations. These problems involve second order differential operators without Fredholm property.
\end{abstract}

Key words: standing waves, non Fredholm operators, embedded solitons, solvability conditions AMS subject classification: $35 \mathrm{P} 30,35 \mathrm{~J} 60$

\section{Introduction}

In the article we consider the following system of $N(N \in \mathbb{N}$ is fixed) nonlocal nonlinear equations in $\mathbb{R}^{d}$ with $1 \leq d \leq 3$, the parameter $\lambda \in \mathbb{R}$, the oscillation frequencies $\omega_{k} \geq 0$ and $1 \leq k \leq N$ :

$i \frac{\partial \psi_{k}}{\partial t}=-\Delta \psi_{k}+\lambda \int_{\mathbb{R}^{d}} G_{k}(x-y)\left[F_{k}\left(\sum_{s=1}^{N}\left|\psi_{s}(y, t)\right|^{2}\right) \psi_{k}(y, t)+U_{k}(y) \psi_{k}(y, t)\right] d y+h_{k}(x) e^{-i \omega_{k} t}$.

Our primary interest in (1.1) is not based on any particular practical applications of this system, but is due to its resemblance to the system of forced, nonlocal, Nonlinear Schrödinger (NLS) equations. Precise conditions on other terms involved in (1.1) will be specified further down. Let us look for a solution of the system above in the form of the $N$ component standing solitary wave

$$
\psi_{k}(x, t)=\phi_{k}(x) e^{-i \omega_{k} t}, \quad 1 \leq k \leq N
$$

The sign under the exponent in (1.2) is negative, which occurs in the case of so-called embedded solitons (see e.g. [5]), as distinct from the standard situation (see e.g. [1], [7]). We substitute (1.2) 
into (1.1) and easily obtain the following system of nonlocal elliptic equations with $1 \leq k \leq N$

$$
-\Delta \phi_{k}-\omega_{k} \phi_{k}+\lambda \int_{\mathbb{R}^{d}} G_{k}(x-y)\left[F_{k}\left(\sum_{s=1}^{N}\left|\phi_{s}(y)\right|^{2}\right) \phi_{k}(y)+U_{k}(y) \phi_{k}(y)\right] d y+h_{k}(x)=0 .
$$

The absolute value $|\lambda|$ here is assumed to be small enough and the solvability conditions for the system of equations (1.3) when $\lambda$ vanishes are given by Lemmas 5 and 6 of [15]. Let us recall that a linear operator acting from a Banach space $E$ into another Banach space $F$ is said to possess the Fredholm property if its image is closed, the dimension of its kernel and the codimension of its image are finite. In our case system (1.3) involves the non Fredholm operators $-\Delta-\omega_{k}: H^{2}\left(\mathbb{R}^{d}\right) \rightarrow L^{2}\left(\mathbb{R}^{d}\right)$. When these operators are considered on $L^{2}\left(\mathbb{R}^{d}\right)$ their essential spectra $\sigma_{\text {ess }}\left(-\Delta-\omega_{k}\right)=\left[-\omega_{k}, \infty\right), 1 \leq k \leq N$ contain the origin. Solvability conditions for nonhomogeneous elliptic problems with operators of that kind were studied extensively in recent years. While linear equations with and without external scalar potentials were covered in the number of works (see e.g. [6], [8]- [12], [14], [15]), nonlinear non Fredholm problems were treated in a few examples as well (see e.g. [2]- [4], [13], [15], [16]). The results for the single equation analogous to system (1.1) were obtained in [15]. Let us denote the inner product of two functions as

$$
\left(f_{1}(x), f_{2}(x)\right)_{L^{2}\left(\mathbb{R}^{d}\right)}:=\int_{\mathbb{R}^{d}} f_{1}(x) \bar{f}_{2}(x) d x,
$$

with a slight abuse of notations when these functions are not square integrable, like those involved in the orthogonality conditions of Theorem 1 below. Indeed, when $f_{1}(x) \in L^{1}\left(\mathbb{R}^{d}\right)$ and $f_{2}(x)$ is bounded, the integral in the right side of the formula above makes sense. The sphere of radius $r>0$ in $\mathbb{R}^{d}$ centered at the origin will be designated as $S_{r}^{d}$. The functional space used in the article will be equipped with the norms

$$
\begin{gathered}
\|u\|_{H^{2}\left(\mathbb{R}^{d}, \mathbb{C}^{N}\right)}^{2}:=\sum_{k=1}^{N}\left\|u_{k}\right\|_{H^{2}\left(\mathbb{R}^{d}\right)}^{2}=\sum_{k=1}^{N}\left\{\left\|u_{k}\right\|_{L^{2}\left(\mathbb{R}^{d}\right)}^{2}+\left\|\Delta u_{k}\right\|_{L^{2}\left(\mathbb{R}^{d}\right)}^{2}\right\}, \\
\|u\|_{L^{2}\left(\mathbb{R}^{d}, \mathbb{C}^{N}\right)}^{2}:=\sum_{k=1}^{N}\left\|u_{k}\right\|_{L^{2}\left(\mathbb{R}^{d}\right)}^{2}
\end{gathered}
$$

for a vector function $u(x):=\left(u_{1}(x), u_{2}(x), \ldots, u_{N}(x)\right) \in H^{2}\left(\mathbb{R}^{d}, \mathbb{C}^{N}\right)$. We will use the closed unit ball centered at the origin in the Sobolev space of vector functions with the norm defined above:

$$
B\left(H^{2}\left(\mathbb{R}^{d}, \mathbb{C}^{N}\right)\right):=\left\{u(x) \in H^{2}\left(\mathbb{R}^{d}, \mathbb{C}^{N}\right) \mid\|u\|_{H^{2}\left(\mathbb{R}^{d}, \mathbb{C}^{N}\right)} \leq 1\right\} .
$$

The norm of a complex-valued vector of $N$ components will be denoted as

$$
|u|_{\mathbb{C}^{N}}^{2}:=\sum_{k=1}^{N}\left|u_{k}\right|^{2}
$$

Our main result is as follows. 
Theorem 1. Let for $1 \leq k \leq N$ the following properties hold: $U_{k}(x) \in L^{\infty}\left(\mathbb{R}^{d}\right)$, the functions $h_{k}(x) \in L^{2}\left(\mathbb{R}^{d}\right)$ and are nontrivial for at least one value of $k, \quad F_{k}(z): \mathbb{R}^{+} \rightarrow \mathbb{R}$ are continuously differentiable, the kernels $G_{k}(x) \in L^{1}\left(\mathbb{R}^{d}\right), 1 \leq d \leq 3$. Let the oscillation frequencies $\omega_{k}>0$ for $1 \leq k \leq M$ and $\omega_{k}=0$ for $M+1 \leq k \leq N$ with a given $1 \leq M \leq N-1, M \in \mathbb{N}$.

I) When the dimension $d=1$ and $1 \leq k \leq M$, let $x G_{k}(x), x h_{k}(x) \in L^{1}(\mathbb{R})$ and

$$
\left(G_{k}(x), \frac{e^{ \pm i \sqrt{\omega_{k}} x}}{\sqrt{2 \pi}}\right)_{L^{2}(\mathbb{R})}=0, \quad\left(h_{k}(x), \frac{e^{ \pm i \sqrt{\omega_{k}} x}}{\sqrt{2 \pi}}\right)_{L^{2}(\mathbb{R})}=0 .
$$

For $M+1 \leq k \leq N$, let $x^{2} G_{k}(x), x^{2} h_{k}(x) \in L^{1}(\mathbb{R})$ and

$$
\left(G_{k}(x), 1\right)_{L^{2}(\mathbb{R})}=0, \quad\left(G_{k}(x), x\right)_{L^{2}(\mathbb{R})}=0, \quad\left(h_{k}(x), 1\right)_{L^{2}(\mathbb{R})}=0, \quad\left(h_{k}(x), x\right)_{L^{2}(\mathbb{R})}=0 .
$$

II) When the dimension $d=2,3$ and $1 \leq k \leq M$, let $x G_{k}(x), x h_{k}(x) \in L^{1}\left(\mathbb{R}^{d}\right)$ and

$$
\left(G_{k}(x), \frac{e^{i p x}}{(2 \pi)^{\frac{d}{2}}}\right)_{L^{2}\left(\mathbb{R}^{d}\right)}=0, \quad\left(h_{k}(x), \frac{e^{i p x}}{(2 \pi)^{\frac{d}{2}}}\right)_{L^{2}\left(\mathbb{R}^{d}\right)}=0 \quad \text { for } \quad p \in S_{\sqrt{\omega_{k}}}^{d} \text { a.e. }
$$

For $M+1 \leq k \leq N$, let $x^{2} h_{k}(x) \in L^{1}\left(\mathbb{R}^{2}\right)$ when $d=2$ and

$$
\left(h_{k}(x), 1\right)_{L^{2}\left(\mathbb{R}^{2}\right)}=0, \quad\left(h_{k}(x), x_{s}\right)_{L^{2}\left(\mathbb{R}^{2}\right)}=0, \quad 1 \leq s \leq 2 .
$$

When $M+1 \leq k \leq N$ and $d=3$ assume $x h_{k}(x) \in L^{1}\left(\mathbb{R}^{3}\right)$ and

$$
\left(h_{k}(x), 1\right)_{L^{2}\left(\mathbb{R}^{3}\right)}=0 .
$$

Moreover, let us assume that for $M+1 \leq k \leq N$ and $d=2,3$ that $x^{2} G_{k}(x) \in L^{1}\left(\mathbb{R}^{d}\right)$ and

$$
\left(G_{k}(x), 1\right)_{L^{2}\left(\mathbb{R}^{d}\right)}=0, \quad\left(G_{k}(x), x_{s}\right)_{L^{2}\left(\mathbb{R}^{d}\right)}=0,1 \leq s \leq d .
$$

Then there exists $\varepsilon>0$ such that for all $\lambda \in \mathbb{R},|\lambda|<\varepsilon$ the system of equations (1.3) possesses a unique nontrivial solution $\phi(x) \in H^{2}\left(\mathbb{R}^{d}, \mathbb{C}^{N}\right)$.

\section{Standing waves of the system of nonlocal, forced equations}

Proof of Theorem 1. Under the assumptions of the theorem stated above by means of the results of Lemmas 5 and 6 of [15] system (1.3) with the vanishing parameter $\lambda$ admits a unique solution $\phi_{0}(x)=\left(\phi_{0,1}(x), \phi_{0,2}(x), \ldots, \phi_{0, N}(x)\right) \in H^{2}\left(\mathbb{R}^{d}, \mathbb{C}^{N}\right), 1 \leq d \leq 3$, such that

$$
-\Delta \phi_{0, k}-\omega_{k} \phi_{0, k}=-h_{k}, \quad 1 \leq k \leq N .
$$

Let us look for the solution of the system of equations (1.3) in the form $\phi(x)=\phi_{0}(x)+\eta(x)$ in the case when $\lambda \neq 0$. For $1 \leq k \leq N$ we easily obtain

$$
-\Delta \eta_{k}-\omega_{k} \eta_{k}+\lambda \int_{\mathbb{R}^{d}} G_{k}(x-y)\left[F_{k}\left(\sum_{s=1}^{N}\left|\phi_{0, s}(y)+\eta_{s}(y)\right|^{2}\right)\left(\phi_{0, k}(y)+\eta_{k}(y)\right)+\right.
$$




$$
\left.+U_{k}(y)\left(\phi_{0, k}(y)+\eta_{k}(y)\right)\right] d y=0 .
$$

For technical purposes we will be using the auxiliary system of equations with $1 \leq k \leq N$

$$
\begin{gathered}
\Delta \xi_{k}+\omega_{k} \xi_{k}=\lambda \int_{\mathbb{R}^{d}} G_{k}(x-y)\left[F_{k}\left(\sum_{s=1}^{N}\left|\phi_{0, s}(y)+\eta_{s}(y)\right|^{2}\right)\left(\phi_{0, k}(y)+\eta_{k}(y)\right)+\right. \\
\left.+U_{k}(y)\left(\phi_{0, k}(y)+\eta_{k}(y)\right)\right] d y
\end{gathered}
$$

Below we will prove that for small enough values of $|\lambda|$ the system of equations (2.1) defines a map $T: B\left(H^{2}\left(\mathbb{R}^{d}, \mathbb{C}^{N}\right)\right) \rightarrow B\left(H^{2}\left(\mathbb{R}^{d}, \mathbb{C}^{N}\right)\right)$. Let us first assume that for a certain $\eta(x) \in$ $B\left(H^{2}\left(\mathbb{R}^{d}, \mathbb{C}^{N}\right)\right)$ there exist two solutions $\xi^{(1),(2)}(x) \in B\left(H^{2}\left(\mathbb{R}^{d}, \mathbb{C}^{N}\right)\right)$ of system (2.1). Clearly, the difference vector function $\xi(x):=\xi^{(1)}(x)-\xi^{(2)}(x) \in H^{2}\left(\mathbb{R}^{d}, \mathbb{C}^{N}\right)$ solves the system of equations

$$
-\Delta \xi_{k}=\omega_{k} \xi_{k}, \quad 1 \leq k \leq N .
$$

But the negative Laplacian considered in the whole space does not have any nontrivial square integrable eigenfunctions. Therefore, $\xi(x)=0$ a.e. in $\mathbb{R}^{d}$.

Let us choose arbitrarily a vector function $\eta(x) \in B\left(H^{2}\left(\mathbb{R}^{d}, \mathbb{C}^{N}\right)\right)$. Evidently, via the Sobolev embedding theorem $\phi_{0, k}(x), \eta_{k}(x) \in L^{\infty}\left(\mathbb{R}^{d}\right), 1 \leq d \leq 3$ for $1 \leq k \leq N$. Therefore, using the assumptions of the theorem as well, we obtain the bounds on the terms of the system of equations (2.1), such that

$$
\left|U_{k}\left(\phi_{0, k}+\eta_{k}\right)\right| \leq\left\|U_{k}\right\|_{L^{\infty}\left(\mathbb{R}^{d}\right)}\left(\left|\phi_{0, k}\right|+\left|\eta_{k}\right|\right) \in L^{2}\left(\mathbb{R}^{d}\right)
$$

and

$$
\begin{gathered}
\left|F_{k}\left(\sum_{s=1}^{N}\left|\phi_{0, s}(y)+\eta_{s}(y)\right|^{2}\right)\left(\phi_{0, k}(y)+\eta_{k}(y)\right)\right| \leq \\
\leq \sup \left|F_{k}(z)\right|_{z \in\left[0, \sum_{s=1}^{N}\left(\left\|\phi_{0, s}\right\|_{L^{\infty}\left(\mathbb{R}^{d}\right)}+\left\|\eta_{s}\right\|_{L^{\infty}\left(\mathbb{R}^{d}\right)}\right)^{2}\right]}\left(\left|\phi_{0, k}(y)\right|+\left|\eta_{k}(y)\right|\right) \in L^{2}\left(\mathbb{R}^{d}\right)
\end{gathered}
$$

as well. We apply the standard Fourier transform (denoted by the "hat" symbol) to the system of equations (2.1) and for $1 \leq k \leq N$ arrive at

$$
\widehat{\xi_{k}}(p)=\lambda(2 \pi)^{\frac{d}{2}} \frac{\widehat{G_{k}}(p)}{\omega_{k}-p^{2}}\left\{\mathcal{F}_{k}(p)+\mathcal{G}_{k}(p)\right\},
$$

with $\mathcal{F}_{k}(p)$ and $\mathcal{G}_{k}(p)$ denoting the transforms of $F_{k}\left(\sum_{s=1}^{N}\left|\phi_{0, s}(y)+\eta_{s}(y)\right|^{2}\right)\left(\phi_{0, k}(y)+\eta_{k}(y)\right)$ and $U_{k}(y)\left(\phi_{0, k}(y)+\eta_{k}(y)\right)$ respectively. Estimates (2.2) and (2.3) imply that $\mathcal{F}_{k}(p), \mathcal{G}_{k}(p) \in L^{2}\left(\mathbb{R}^{d}\right)$. Obviously

$$
p^{2} \widehat{\xi_{k}}(p)=\lambda(2 \pi)^{\frac{d}{2}} \frac{p^{2} \widehat{G_{k}}(p)}{\omega_{k}-p^{2}}\left\{\mathcal{F}_{k}(p)+\mathcal{G}_{k}(p)\right\}
$$

For technical purposes we will be using the following quantities for $1 \leq k \leq N$, namely

$$
N_{\omega_{k}, d}:=\max \left\{\left\|\frac{\widehat{G}_{k}(p)}{\omega_{k}-p^{2}}\right\|_{L^{\infty}\left(\mathbb{R}^{d}\right)}, \quad\left\|\frac{p^{2} \widehat{G}_{k}(p)}{\omega_{k}-p^{2}}\right\|_{L^{\infty}\left(\mathbb{R}^{d}\right)}\right\}, \omega_{k} \geq 0,1 \leq d \leq 3 .
$$


Orthogonality conditions (1.5), (1.6), (1.7) and (1.10) along with Lemmas A1 and A2 of [13] imply that for $1 \leq k \leq N$ we have $N_{\omega_{k}, d}<\infty$. Hence,

$$
\begin{gathered}
\left|\widehat{\xi_{k}}(p)\right| \leq|\lambda|(2 \pi)^{\frac{d}{2}} N_{\omega_{k}, d}\left\{\left|\mathcal{F}_{k}(p)\right|+\left|\mathcal{G}_{k}(p)\right|\right\} \in L^{2}\left(\mathbb{R}^{d}\right), \\
\left|p^{2} \widehat{\xi_{k}}(p)\right| \leq|\lambda|(2 \pi)^{\frac{d}{2}} N_{\omega_{k}, d}\left\{\left|\mathcal{F}_{k}(p)\right|+\left|\mathcal{G}_{k}(p)\right|\right\} \in L^{2}\left(\mathbb{R}^{d}\right)
\end{gathered}
$$

Therefore, we easily obtain the upper bound on the norm

$$
\begin{gathered}
\|\xi\|_{H^{2}\left(\mathbb{R}^{d}, \mathbb{C}^{N}\right)}^{2}=\sum_{k=1}^{N}\left\{\left\|\widehat{\xi_{k}}(p)\right\|_{L^{2}\left(\mathbb{R}^{d}\right)}^{2}+\left\|p^{2} \widehat{\xi_{k}}(p)\right\|_{L^{2}\left(\mathbb{R}^{d}\right)}^{2}\right\} \leq \\
\leq 2 \lambda^{2}(2 \pi)^{d} \sum_{k=1}^{N} N_{\omega_{k}, d}^{2}\left\|\left|\mathcal{F}_{k}(p)\right|+\left|\mathcal{G}_{k}(p)\right|\right\|_{L^{2}\left(\mathbb{R}^{d}\right)}^{2} .
\end{gathered}
$$

Thus $\xi \in B\left(H^{2}\left(\mathbb{R}^{d}, \mathbb{C}^{N}\right)\right)$ for all the values of the parameter $|\lambda|$ sufficiently small and $T \eta=$ $\xi$. Finally, we need to show that the map $T: B\left(H^{2}\left(\mathbb{R}^{d}, \mathbb{C}^{N}\right)\right) \rightarrow B\left(H^{2}\left(\mathbb{R}^{d}, \mathbb{C}^{N}\right)\right)$ defined by system (2.1) is a strict contraction. To achieve this goal, let us choose arbitrarily $\eta_{1}(x), \eta_{2}(x) \in$ $B\left(H^{2}\left(\mathbb{R}^{d}, \mathbb{C}^{N}\right)\right)$. Evidently $T \eta_{1,2}=\xi_{1,2} \in B\left(H^{2}\left(\mathbb{R}^{d}, \mathbb{C}^{N}\right)\right)$ via the system of equations (2.1) when $|\lambda|$ is small enough. Obviously for $1 \leq k \leq N$

$$
\widehat{\xi_{1, k}}(p)-\widehat{\xi_{2, k}}(p)=\lambda(2 \pi)^{\frac{d}{2}} \frac{\widehat{G_{k}}(p)}{\omega_{k}-p^{2}}\left\{\mathcal{F}_{1, k}(p)-\mathcal{F}_{2, k}(p)+\mathcal{G}_{1, k}(p)-\mathcal{G}_{2, k}(p)\right\} .
$$

Here $\mathcal{F}_{j, k}(p)$ and $\mathcal{G}_{j, k}(p)$ denote the Fourier images of expressions

$$
F_{k}\left(\sum_{s=1}^{N}\left|\phi_{0, s}(x)+\eta_{j, s}(x)\right|^{2}\right)\left(\phi_{0, k}(x)+\eta_{j, k}(x)\right) \quad \text { and } \quad U_{k}(x)\left(\phi_{0, k}(x)+\eta_{j, k}(x)\right)
$$

respectively with $j=1,2$. Thus

$$
p^{2} \widehat{\xi_{1, k}}(p)-p^{2} \widehat{\xi_{2, k}}(p)=\lambda(2 \pi)^{\frac{d}{2}} \frac{p^{2} \widehat{G_{k}}(p)}{\omega_{k}-p^{2}}\left\{\mathcal{F}_{1, k}(p)-\mathcal{F}_{2, k}(p)+\mathcal{G}_{1, k}(p)-\mathcal{G}_{2, k}(p)\right\}
$$

We easily arrive at the upper bound for $\left\|\xi_{1, k}(x)-\xi_{2, k}(x)\right\|_{L^{2}\left(\mathbb{R}^{d}\right)}$ as

$$
\begin{gathered}
|\lambda|(2 \pi)^{\frac{d}{2}} N_{\omega_{k}, d}\left\{\left\|F_{k}\left(\sum_{s=1}^{N}\left|\phi_{0, s}+\eta_{1, s}\right|^{2}\right)\left(\phi_{0, k}+\eta_{1, k}\right)-F_{k}\left(\sum_{s=1}^{N}\left|\phi_{0, s}+\eta_{2, s}\right|^{2}\right)\left(\phi_{0, k}+\eta_{2, k}\right)\right\|_{L^{2}\left(\mathbb{R}^{d}\right)}+\right. \\
\left.+\left\|U_{k}\left(\eta_{1, k}-\eta_{2, k}\right)\right\|_{L^{2}\left(\mathbb{R}^{d}\right)}\right\} .
\end{gathered}
$$

The analogical estimate from above is valid for $\left\|\Delta \xi_{1, k}(x)-\Delta \xi_{2, k}(x)\right\|_{L^{2}\left(\mathbb{R}^{d}\right)}$. Obviously

$$
\left\|U_{k}\left(\eta_{1, k}-\eta_{2, k}\right)\right\|_{L^{2}\left(\mathbb{R}^{d}\right)} \leq\left\|U_{k}\right\|_{L^{\infty}\left(\mathbb{R}^{d}\right)}\left\|\eta_{1, k}-\eta_{2, k}\right\|_{L^{2}\left(\mathbb{R}^{d}\right)} .
$$


We express

$$
\begin{gathered}
F_{k}\left(\sum_{s=1}^{N}\left|\phi_{0, s}+\eta_{1, s}\right|^{2}\right)\left(\phi_{0, k}+\eta_{1, k}\right)-F_{k}\left(\sum_{s=1}^{N}\left|\phi_{0, s}+\eta_{2, s}\right|^{2}\right)\left(\phi_{0, k}+\eta_{2, k}\right)= \\
=\left\{F_{k}\left(\sum_{s=1}^{N}\left|\phi_{0, s}+\eta_{1, s}\right|^{2}\right)-F_{k}\left(\sum_{s=1}^{N}\left|\phi_{0, s}+\eta_{2, s}\right|^{2}\right)\right\}\left(\phi_{0, k}+\eta_{2, k}\right)+ \\
+F_{k}\left(\sum_{s=1}^{N}\left|\phi_{0, s}+\eta_{1, s}\right|^{2}\right)\left(\eta_{1, k}-\eta_{2, k}\right) .
\end{gathered}
$$

The Sobolev embedding theorem applied in $\mathbb{R}^{d}$ with $1 \leq d \leq 3$ yields

$$
\left|\phi_{0, s}+\eta_{j, s}\right| \leq c_{e}\left(\left\|\phi_{0, s}\right\|_{H^{2}\left(\mathbb{R}^{d}\right)}+\left\|\eta_{j, s}\right\|_{H^{2}\left(\mathbb{R}^{d}\right)}\right),
$$

where $c_{e}$ denotes the constant of the embedding, $1 \leq s \leq N$ and $j=1,2$. Hence, we arrive at

$$
\sum_{s=1}^{N}\left|\phi_{0, s}+\eta_{j, s}\right|^{2} \leq 2 c_{e}^{2}\left(1+\left\|\phi_{0}\right\|_{H^{2}\left(\mathbb{R}^{d}, \mathbb{C}^{N}\right)}^{2}\right), \quad j=1,2,
$$

such that

$$
\left\|F_{k}\left(\sum_{s=1}^{N}\left|\phi_{0, s}+\eta_{1, s}\right|^{2}\right)\left(\eta_{1, k}-\eta_{2, k}\right)\right\|_{L^{2}\left(\mathbb{R}^{d}\right)} \leq \sup \left|F_{k}(z)\right|_{z \in\left[0,2 c_{e}^{2}\left(1+\left\|\phi_{0}\right\|_{H^{2}\left(\mathbb{R}^{d}, \mathbb{C}^{N}\right)}^{2}\right)\right]}\left\|\eta_{1, k}-\eta_{2, k}\right\|_{L^{2}\left(\mathbb{R}^{d}\right)} .
$$

Let us make use of the identity

$$
F_{k}\left(\sum_{s=1}^{N}\left|\phi_{0, s}+\eta_{1, s}\right|^{2}\right)-F_{k}\left(\sum_{s=1}^{N}\left|\phi_{0, s}+\eta_{2, s}\right|^{2}\right)=\int_{\sum_{s=1}^{N}\left|\phi_{0, s}+\eta_{2, s}\right|^{2}}^{\sum_{s=1}^{N}\left|\phi_{0, s}+\eta_{1, s}\right|^{2}} F_{k}^{\prime}(z) d z .
$$

For technical purposes via the Sobolev embedding theorem we estimate

$$
\begin{gathered}
\left|\sum_{s=1}^{N}\right| \phi_{0, s}+\left.\eta_{1, s}\right|^{2}-\sum_{s=1}^{N}\left|\phi_{0, s}+\eta_{2, s}\right|^{2}\left|\leq 2 c_{e} \sum_{s=1}^{N}\right| \eta_{1, s}-\eta_{2, s} \mid\left(1+\left\|\phi_{0, s}\right\|_{H^{2}\left(\mathbb{R}^{d}\right)}\right) \leq \\
\leq 2 c_{e} \sqrt{\sum_{s=1}^{N}\left|\eta_{1, s}-\eta_{2, s}\right|^{2}} \sqrt{\sum_{l=1}^{N}\left(1+\left\|\phi_{0, l}\right\|_{H^{2}\left(\mathbb{R}^{d}\right)}\right)^{2}} \leq 2 \sqrt{2} c_{e}\left|\eta_{1}-\eta_{2}\right|_{\mathbb{C}^{N}} \sqrt{N+\left\|\phi_{0}\right\|_{H^{2}\left(\mathbb{R}^{d}, \mathbb{C}^{N}\right)}^{2}},
\end{gathered}
$$

which enables us to obtain

$$
\left|\left\{F_{k}\left(\sum_{s=1}^{N}\left|\phi_{0, s}+\eta_{1, s}\right|^{2}\right)-F_{k}\left(\sum_{s=1}^{N}\left|\phi_{0, s}+\eta_{2, s}\right|^{2}\right)\right\}\left(\phi_{0, k}+\eta_{2, k}\right)\right| \leq 2 \sqrt{2} c_{e}^{2} \sqrt{N+\left\|\phi_{0}\right\|_{H^{2}\left(\mathbb{R}^{d}, \mathbb{C}^{N}\right)}^{2} \times}
$$




$$
\times \sup \left|F_{k}^{\prime}(z)\right|_{z \in\left[0,2 c_{e}^{2}\left(1+\left\|\phi_{0}\right\|_{H^{2}\left(\mathbb{R}^{d}, \mathbb{C}^{N}\right)}^{2}\right)\right.}\left(\left\|\phi_{0, k}\right\|_{H^{2}\left(\mathbb{R}^{d}\right)}+\left\|\eta_{2, k}\right\|_{H^{2}\left(\mathbb{R}^{d}\right)}\right)\left|\eta_{1}-\eta_{2}\right|_{\mathbb{C}^{N}} .
$$

Therefore, we manage to derive the upper bound

$$
\begin{gathered}
\left\|\left\{F_{k}\left(\sum_{s=1}^{N}\left|\phi_{0, s}+\eta_{1, s}\right|^{2}\right)-F_{k}\left(\sum_{s=1}^{N}\left|\phi_{0, s}+\eta_{2, s}\right|^{2}\right)\right\}\left(\phi_{0, k}+\eta_{2, k}\right)\right\|_{L^{2}\left(\mathbb{R}^{d}\right)} \leq \\
\leq 2 \sqrt{2} c_{e}^{2} \sqrt{N+\left\|\phi_{0}\right\|_{H^{2}\left(\mathbb{R}^{d}, \mathbb{C}^{N}\right)}^{2}} \sup \left|F_{k}^{\prime}(z)\right|_{z \in\left[0,2 c_{e}^{2}\left(1+\left\|\phi_{0}\right\|_{H^{2}\left(\mathbb{R}^{d}, \mathbb{C}^{N}\right)}^{2}\right)\right]} \times \\
\times\left(\left\|\phi_{0, k}\right\|_{H^{2}\left(\mathbb{R}^{d}\right)}+\left\|\eta_{2, k}\right\|_{H^{2}\left(\mathbb{R}^{d}\right)}\right)\left\|\eta_{1}-\eta_{2}\right\|_{L^{2}\left(\mathbb{R}^{d}, \mathbb{C}^{N}\right)} .
\end{gathered}
$$

Collecting the estimates obtained above we arrive at

$$
\begin{aligned}
& \left\|\xi_{1, k}-\xi_{2, k}\right\|_{L^{2}\left(\mathbb{R}^{d}\right)} \leq|\lambda|(2 \pi)^{\frac{d}{2}} N_{\omega_{k}, d}\left\{\left\|U_{k}\right\|_{L^{\infty}\left(\mathbb{R}^{d}\right)}+\sup \left|F_{k}(z)\right|_{z \in\left[0,2 c_{e}^{2}\left(1+\left\|\phi_{0}\right\|_{H^{2}\left(\mathbb{R}^{d}, \mathbb{C}^{N}\right)}^{2}\right)\right]}+2 \sqrt{2} c_{e}^{2} \times\right. \\
& \left.\times \sqrt{N+\left\|\phi_{0}\right\|_{H^{2}\left(\mathbb{R}^{d}, \mathbb{C}^{N}\right)}^{2}}\left(1+\left\|\phi_{0}\right\|_{H^{2}\left(\mathbb{R}^{d}, \mathbb{C}^{N}\right)}\right) \sup \left|F_{k}^{\prime}(z)\right|_{z \in\left[0,2 c_{e}^{2}\left(1+\left\|\phi_{0}\right\|_{H^{2}\left(\mathbb{R}^{d}, \mathbb{C}^{N}\right)}^{2}\right)\right]}\right\}\left\|\eta_{1}-\eta_{2}\right\|_{L^{2}\left(\mathbb{R}^{d}, \mathbb{C}^{N}\right)} .
\end{aligned}
$$

Finally, we have

$$
\begin{aligned}
&\left\|T \eta_{1}-T \eta_{2}\right\|_{H^{2}\left(\mathbb{R}^{d}, \mathbb{C}^{N}\right)} \leq \sqrt{2}(2 \pi)^{\frac{d}{2}}|\lambda|\left[\sum _ { k = 1 } ^ { N } N _ { \omega _ { k } , d } ^ { 2 } \left\{\left\|U_{k}\right\|_{L^{\infty}\left(\mathbb{R}^{d}\right)}+\sup \left|F_{k}(z)\right|_{z \in\left[0,2 c_{e}^{2}\left(1+\left\|\phi_{0}\right\|_{H^{2}\left(\mathbb{R}^{d}, \mathbb{C}^{N}\right)}^{2}\right)\right]+}\right.\right. \\
&+2 \sqrt{2} c_{e}^{2} \sqrt{N+\left\|\phi_{0}\right\|_{H^{2}\left(\mathbb{R}^{d}, \mathbb{C}^{N}\right)}^{2}}(1+\left.\left.\left.\left\|\phi_{0}\right\|_{H^{2}\left(\mathbb{R}^{d}, \mathbb{C}^{N}\right)}\right) \sup \left|F_{k}^{\prime}(z)\right|_{z \in\left[0,2 c_{e}^{2}\left(1+\left\|\phi_{0}\right\|_{H^{2}\left(\mathbb{R}^{d}, \mathbb{C}^{N}\right)}^{2}\right)\right]}\right\}^{2}\right]^{\frac{1}{2}} \times \\
& \times\left\|\eta_{1}-\eta_{2}\right\|_{H^{2}\left(\mathbb{R}^{d}, \mathbb{C}^{N}\right)} .
\end{aligned}
$$

Therefore, for all the values of the parameter $|\lambda|$ sufficiently small, the map $T: B\left(H^{2}\left(\mathbb{R}^{d}, \mathbb{C}^{N}\right)\right) \rightarrow$ $B\left(H^{2}\left(\mathbb{R}^{d}, \mathbb{C}^{N}\right)\right)$ generated by system $(2.1)$ is a strict contraction, such that it possesses a unique fixed point $\eta \in B\left(H^{2}\left(\mathbb{R}^{d}, \mathbb{C}^{N}\right)\right)$. The unique solution of system (1.3) belonging to $H^{2}\left(\mathbb{R}^{d}, \mathbb{C}^{N}\right)$ is nontrivial by means of our assumption that for at least one value of $1 \leq k \leq N$ the function $h_{k}(x)$ does not vanish in $\mathbb{R}^{d}$.

\section{References}

[1] S. Cuccagna, D. Pelinovsky, V. Vougalter. Spectra of positive and negative energies in the linearized NLS problem. Comm. Pure Appl. Math., 58 (2005), No.1, 1-29.

[2] A. Ducrot, M. Marion, V. Volpert. Systemes de réaction-diffusion sans propriété de Fredholm. C.R.Math.Acad.Sci.Paris, 340 (2005), No. 9, 659-664.

[3] A. Ducrot, M. Marion, V. Volpert: Reaction-diffusion problems with non Fredholm operators. Adv. Differential Equations, 13 (2008), No. 11-12, 1151-1192. 
[4] A. Ducrot, M. Marion and V. Volpert: Reaction- diffusion waves (with the Lewis number different from 1). Publibook, Paris, 2009.

[5] D.E. Pelinovsky, J. Yang. A normal form for nonlinear resonance of embedded solitons. R. Soc. Lond. Proc. Ser. A Math. Phys. Eng. Sci. 458 (2002), No. 2022, 1469-1497.

[6] V. Volpert, B. Kazmierczak, M. Massot, Z.Peradzynski. Solvability conditions for elliptic problems with non-Fredholm operators. Appl. Math., 29 (2002), No. 2, 219-238.

[7] V. Vougalter. On threshold eigenvalues and resonances for the linearized NLS equation. Math. Model. Nat. Phenom. 5 (2010), No.4, 448-469.

[8] V. Vougalter, V. Volpert. Solvability conditions for some non Fredholm operators. Proc. Edinb. Math. Soc. (2), 54 (2011), No. 1, 249-271.

[9] V. Vougalter, V. Volpert. On the solvability conditions for some non Fredholm operators. Int. J. Pure Appl. Math., 60 (2010), No. 2, 169-191.

[10] V. Vougalter, V. Volpert. On the solvability conditions for the diffusion equation with convection terms. Commun. Pure Appl. Anal., 11 (2012), No.1, 365-373.

[11] V. Vougalter, V. Volpert. Solvability relations for some non Fredholm operators. Int. Electron. J. Pure Appl. Math., 2 (2010), No. 1, 75-83.

[12] V. Volpert, V. Vougalter. On the solvability conditions for a linearized Cahn-Hilliard equation. Rend. Istit. Mat. Univ. Trieste, 43 (2011), 1-9.

[13] V. Vougalter, V. Volpert. On the existence of stationary solutions for some non-Fredholm integro-differential equations. Doc. Math., 16 (2011), 561-580.

[14] V. Vougalter, V. Volpert. Solvability conditions for a linearized Cahn-Hilliard equation of sixth order. Math. Model. Nat. Phenom., 7 (2012), No. 2, 146-154.

[15] V. Vougalter, V. Volpert. Solvability conditions for some linear and nonlinear non-Fredholm elliptic problems. To appear in Anal. Math. Phys.

[16] V. Volpert, V. Vougalter. On the existence of stationary solutions for some systems of nonFredholm integro-differential equations. Disc., Nonlin. and Complex., 1 (2012), No.2, 85-98. 